\title{
A artéria celíaca em Didelphis albiventris (gambá)
}

\author{
The celiac artery in Didelphis albiventris (opossum) \\ Paulete de Oliveira Vargas Culau',2, Sueli Hoff Reckziegel ${ }^{2}$, Laura Ver Goltz² \& Ana Cristina Pacheco de \\ Araújo $^{3}$
}

\begin{abstract}
Background: This member of the Didelphidea family, commonly known as opossum, is widely distributed in the American territory, with species found from southern Canada up to northern Argentina. Similarly to all marsupials, they are characterized by a short gestational period, followed by a long development period. Opossums are arboreal, terrestrial slow animals of lonely and nocturnal habits. They are omnivore animals, eating from small rodents, birds, eggs and amphibians to fruits and vegetables. These general characteristics have drawn in the interest and curiosity of the scientific community to this animal, which is now the object of study in several knowledge areas. In order to find morphological information that could assist in discussions from a functional point of view and that could offer support for measures that aim at protecting opossums in their natural environment, the objective of the present study is to divulge the anatomical behavior of their celiac artery and its branches, including its distribution areas, considering the importance of these vessels in the blood supply of several digestive organs.

Materials, Methods \& Results: In this study, the celiac artery of 24 opossums (Didelphis albiventris), of which 17 were females and 7 were males, was systematized. For the purpose, their arterial system was filled with colored latex 603 . The abdominal aorta gave off its first visceral collateral branch, the celiac-mesenteric trunk, which originated the celiac artery and the cranial mesenteric artery in $87.5 \%$ of the samples, but in $12.5 \%$ of the samples, the abdominal aorta gave off these arteries individually. The celiac artery gave off the lienal and hepatic arteries. The lienal artery gave off the left gastric artery to the lesser curvature of the stomach, supplying its parietal and visceral side, also giving off esophageal branches. The lienal artery reached the splenic hilum, giving off several pancreatic branches during its path. Once it reached the splenic hilum, it gave off its own lienal branches and then continued as left gastroepiploic artery towards the greater stomach curvature. The hepatic artery was projected cranially, giving off the gastroduodenal artery and cranial pancreaticoduodenal artery reaching the portal fissure, where hepatic branches arose towards the liver. The gastroduodenal artery gave off the right gastroepiploic artery towards the greater curvature of the stomach, also giving off the right gastric artery towards the lesser curvature of the stomach and then became divided into one branch to the visceral side and one branch to the parietal side, also giving off esophageal and pyloric branches. The cranial pancreaticoduodenal artery branched to the cranial duodenum and right lobe of the pancreas.

Discussion: In the majority of opossums (87.5\%), the celiac artery had origin in a common trunk with the cranial mesenteric artery, but in $12.5 \%$ of the animals, this origin took place separately. In opossums (Didelphis albiventris), regardless of its origin, the celiac artery was a single vessel and, essentially, it was divided into the hepatic artery and the lienal artery in $100 \%$ of the samples. The left gastric artery arose from the lienal artery, next to the origin of this latter in the celiac artery, similarly to the findings in the collared peccary, swine and equine. Therefore, the celiac artery, through its branches, was the artery responsible for the blood supply of the stomach, pancreas, liver, spleen and first part of the duodenum.
\end{abstract}

Keywords: abdominal aorta, abdominal vascularization, marsupial.

Descritores: aorta abdominal, vascularização abdominal, marsupial. 


\section{INTRODUÇÃO}

Membros da família Didelphidea, comumente chamados gambás, apresentam-se distribuídos ao longo do território americano, com espécies que cobrem desde o sul do Canadá até o norte da Argentina. Como todos os marsupiais, caracterizam-se por apresentar um curto período de gestação seguido de um longo período de desenvolvimento. O gambá é um animal lento, solitário, arborícola e terrestre e de hábito noturno. São onívoros, devorando pequenos roedores, aves, ovos, anfíbios, frutas e vegetais. Estas características gerais despertaram o interesse e a curiosidade da comunidade científica sobre este animal, tornando-o objeto de estudo nas mais diversas áreas do conhecimento [7].

Quanto aos aspectos relativos ao comportamento anatômico da artéria celíaca no gambá, nenhuma informação foi obtida na literatura consultada, tornando oportunas, portanto, abordagens referentes a outras espécies selvagens, como o ratão-dobanhado (Myocastor coypus) [3,9,10], o ouriçocacheiro (Sphiggurus spp.) [8], o queixada (Tayassu pecan) e o cateto (Tayassu tajacu) [2]. Reportando-se às espécies domésticas, foram utilizados o cão, o porco e o cavalo $[1,4,5]$.

Na busca de informações de cunho morfológico que possam subsidiar discussões do ponto de vista funcional, resultando em subsídios para melhor compreensão da fisiologia desses animais e, por consequência, oferecer suporte para medidas que visem a protegê-los no seu ambiente natural, o presente trabalho tem como objetivo divulgar o comportamento anatômico da artéria celíaca e seus ramos, incluindo os territórios de sua distribuição, haja vista a importância desses vasos na irrigação sanguínea de diversos órgãos do aparelho digestivo.

\section{MATERIAIS E MÉTODOS}

Utilizaram-se 24 gambás (Didelphis albiventris), 17 fêmeas e sete machos, jovens e adultos, capturados em municípios do estado do Rio Grande do Sul, sob licença $n^{\circ}$ 079/98 e 270/99, processo $n^{\circ} 1887 /$ 98-99, do Instituto Brasileiro do Meio Ambiente e Recursos Renováveis - IBAMA.

Os animais foram previamente tranquilizados com éter sulfúrico, anestesiados com tiopental ${ }^{1}$ e receberam 2500 UI de heparina ${ }^{2}$ via intraperitonial. Abriu-se a caixa torácica ventralmente em plastrão, canulou-se a artéria aorta torácica próximo ao diafragma e o sistema foi lavado com solução salina. Após, preencheu-se o sistema com látex 603, corado em vermelho (BASF), permanecendo em água corrente por uma hora para solidificação do mesmo. Rebateu-se a pele e fez-se injeção intraperitoneal de $150 \mathrm{ml}$ de formaldeído a $20 \%$, sendo o animal imerso nesta solução por sete dias.

Abriu-se a cavidade abdominal na linha mediana ventral, as artérias foram dissecadas, desenhos esquemáticos de todas as peças foram confeccionados e algumas amostras fotografadas para documentação. As designações seguiram a Nômina Anatômica Veterinária [6].

\section{RESULTADOS}

Observando o comportamento anatômico da artéria celíaca em 24 gambás (Didelphis albiventris), verificou-se que a sua origem ocorreu na face ventral da aorta abdominal, imediatamente caudal ao hiato aórtico, em sua maioria $(87,5 \%)$ em tronco comum com a artéria mesentérica cranial (Figuras 1, 2 e 3), ou separadamente $(12,5 \%)$.

Independentemente de sua origem, a artéria celíaca foi um vaso ímpar que se dividiu nas artérias hepática e lienal em 100\% dos exemplares (Figuras $1,2$ e 3$)$.

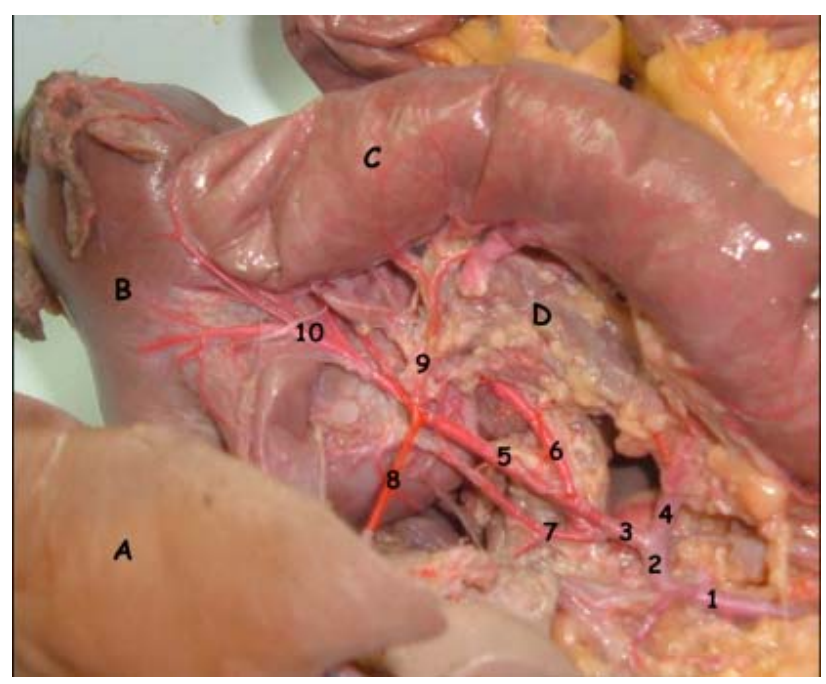

Figura 1. Vista lateral direita da cavidade abdominal de Didelphis albiventris (gambá) para salientar a origem e distribuição da artéria celíaca.1- aorta abdominal; 2- tronco celíaco-mesentérico; 3- a. celíaca; 4- a. mesentérica cranial; 5- a. hepática; 6- a. lienal; 7- a. gástrica esquerda; 8- a. hepática própria; 9- a. pancreaticoduodenal cranial; 10- a. gástrica direita; A- fígado; B- estômago; C- duodeno; D- pâncreas. 
A artéria lienal foi um dos ramos terminais da artéria celíaca (Figura 2). Ela irrigou o baço, a curvatura maior e paredes adjacentes do estômago, omento maior e lobo esquerdo do pâncreas. A artéria lienal emitiu a artéria gástrica esquerda e dirigiu-se para a esquerda relacionando-se com o lobo pancreático esquerdo, enviando ramos pancreáticos. Deste ponto, ela seguiu no sentido da superfície visceral do estômago estendendo-se ventralmente ao longo do ligamento gastrolienal, emitindo vários ramos lienais próprios para o hilo do baço, continuando-se como artéria gastroepiplóica esquerda. Esta percorreu a curvatura maior do estômago, enviando numerosas e delgadas artérias gástricas curtas emitindo simultaneamente ramos epiplóicos. Esta artéria terminou em anastomose com a artéria gastroepiplóica direita.

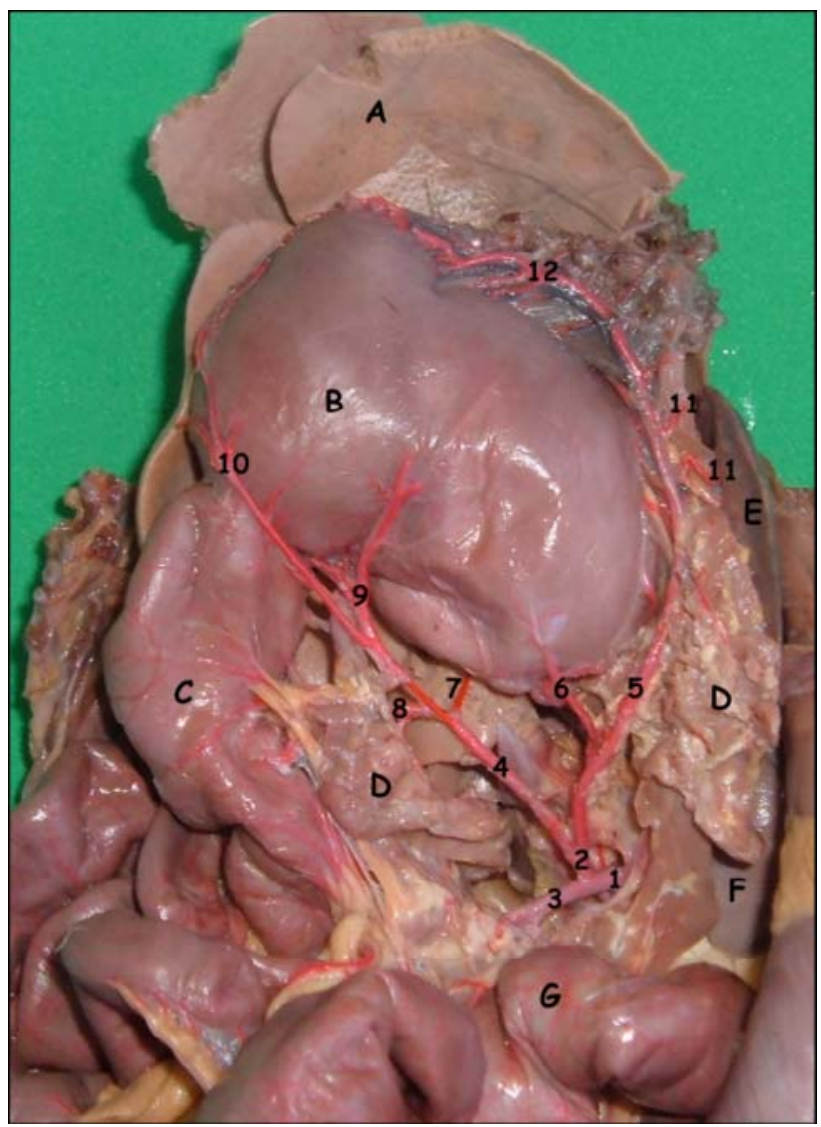

Figura 2. Vista ventral da cavidade abdominal de Didelphis albiventris (gambá) para salientar a origem e distribuição da artéria celíaca. 1- tronco celíaco-mesentérico; 2- a. celíaca; 3- a. mesentérica cranial; 4- a. hepática; 5- a. lienal; 6- a. gástrica esquerda; 7- a. hepática própria; 8- a. pancreaticoduodenal cranial; 9- a. gástrica direita; 10gastroepiplóica direita; 11 - a. lienal própria; 12- a. gastroepiplóica esquerda; A- fígado; B- estômago; Cduodeno; D- pâncreas; E- baço; F- rim esquerdo; G- jejunoíleo.
A artéria gástrica esquerda surgiu da artéria lienal, próximo à origem desta última na artéria celíaca (Figura 2). Orientou-se para a curvatura menor do estômago, lançando ramos esofágicos, ramos para a curvatura maior do estômago, extremidade esquerda e terminou dividindo-se dorsal e caudalmente ao cárdia, nos ramos parietal e visceral. O ramo parietal cruzou a curvatura menor à direita do cárdia e ramificou-se na superfície parietal do estômago. $\mathrm{O}$ ramo visceral foi distribuído de modo semelhante ao ramo parietal, na superfície visceral do estômago.

A artéria hepática projetou-se cranialmente, emitindo como seu primeiro ramo a artéria gastroduodenal em $62,5 \%$ dos casos e a artéria pancreaticoduodenal cranial em 37,5\%. A artéria hepática, ao atingir a cisura portal, emitiu ramos hepáticos próprios para o fígado. A artéria gastroduodenal lançou a artéria gastroepiplóica direita que alcançou o piloro e distribuiu-se para a curvatura maior do estômago anastomosando-se com a artéria gastroepiplóica esquerda, ramo da artéria lienal (Figura 2). Emitiu também a artéria gástrica direita que se dirigiu para a curvatura menor do estômago dividindo-se em um ramo para a face visceral e um ramo para a face parietal, emitindo também ramos esofágicos e pilóricos (Figuras 1, 2 e 3). A artéria pancreaticoduodenal cranial distribuiu-se para a porção inicial do duodeno e lobo direito do pâncreas.

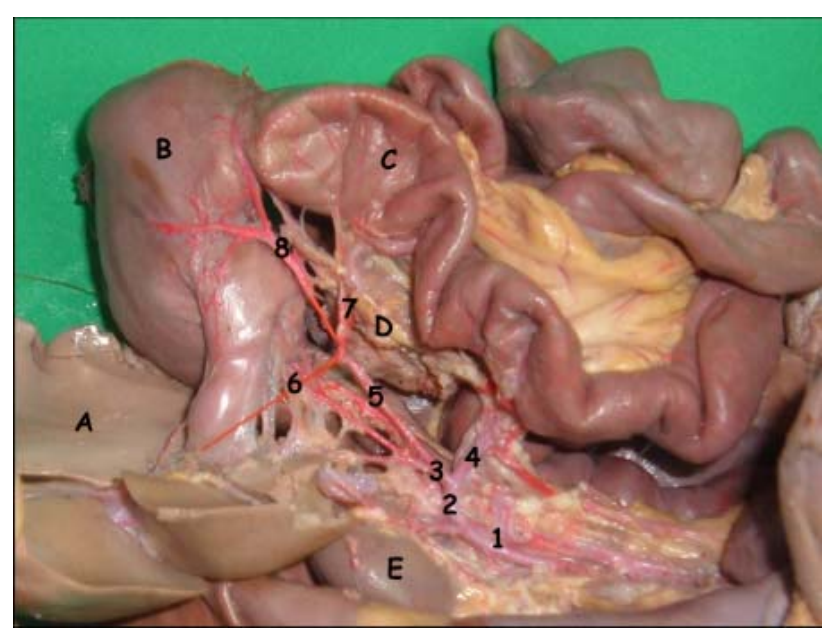

Figura 3. Vista lateral direita da cavidade abdominal de Didelphis albiventris (gambá) para salientar a origem e distribuição da artéria celíaca.1- aorta abdominal; 2- tronco celíaco-mesentérico; 3- a. celíaca; 4- a. mesentérica cranial; 5- a. hepática; 6- a. hepática própria; 7- a. pancreaticoduodenal cranial; 8- a. gástrica direita; A- fígado; B- estômago; C- duodeno; D- pâncreas; E- rim direito. 


\section{DISCUSSÃO}

Quanto aos aspectos relativos ao comportamento anatômico da artéria celíaca no gambá, nenhuma informação foi obtida na literatura consultada, tornando oportunas, portanto, abordagens referentes a outras espécies selvagens.

No ratão-do-banhado (Myocastor coypus), a artéria celíaca originou-se isoladamente $(70 \%)$ da face ventral da aorta abdominal, próximo ao hiato aórtico, ou em tronco comum com a artéria mesentérica cranial $(30 \%)[9,10]$. Em outro trabalho com ratãodo-banhado (Myocastor coypus) ou nutria, a aorta abdominal emitiu em sequência a artéria celíaca e a artéria mesentérica cranial, ventralmente e justapostas [3]. Por outro lado, no ouriço-cacheiro (Sphiggurus spp.) [8], no queixada (Tayassu pecan) e no cateto (Tayassu tajacu) a artéria celíaca mostrou-se única, originando-se da face ventral da aorta abdominal [2]. Em nosso trabalho, no gambá, a artéria celíaca originou-se em sua maioria $(87,5 \%)$ em tronco comum com a artéria mesentérica cranial, ou separadamente $(12,5 \%)$.

As divisões primárias da artéria celíaca, as artérias lienal, gástrica esquerda e hepática surgiram por trifurcação (60\%) ou bifurcação (40\%), neste caso, a artéria lienal achou-se separada de um tronco comum formado pelas duas outras no ratão-do-banhado [10]. No ouriço-cacheiro (Sphiggurus spp.), seus ramos primários foram as artérias gástrica esquerda, lienal e hepática [8]. No queixada (Tayassu pecan), a trifurcação da artéria celíaca, originando as artérias lienal, gástrica esquerda e hepática ocorreu com maior frequência $(71,41 \%)$, enquanto no cateto (Tayassu tajacu) o referido vaso originou principalmente $(80 \%)$ um tronco gastrolienal e a artéria hepática individualmente [2].

Reportando-se às espécies domésticas, no cão a artéria celíaca originou as artérias gástrica esquerda, lienal e hepática, enquanto no suíno e no equino emitiu as artérias hepática e lienal, sendo que a artéria gástrica esquerda tem origem comum com a artéria lienal $[1,4,5]$.
No gambá (Didelphis albiventris), independentemente de sua origem, a artéria celíaca foi um vaso ímpar e essencialmente dividiu-se nas artérias hepática e lienal em $100 \%$ dos exemplares. A artéria gástrica esquerda surgiu da artéria lienal, próximo à origem desta última na artéria celíaca, à semelhança do que foi encontrado no cateto, no suíno e no equino.

Em relação à área de vascularização da artéria celíaca no gambá, esta se manteve semelhante aos outros animais, abrangendo o estômago, baço, fígado, pâncreas e porção inicial do duodeno.

\section{CONCLUSÕES}

A aorta abdominal emitiu o seu primeiro ramo colateral visceral, o tronco celíaco-mesentérico que deu origem à artéria celíaca e a artéria mesentérica cranial em $87,5 \%$ das peças, e emitiu isoladamente estas artérias em 12,5\%.

A artéria celíaca emitiu as artérias lienal e hepática. A artéria gástrica esquerda originou-se da artéria lienal.

A artéria lienal emitiu a artéria gástrica esquerda para a curvatura menor do estômago e esôfago, ramos pancreáticos, ramos lienais próprios e continuou-se como artéria gastroepiplóica esquerda para a curvatura maior do estômago.

A artéria hepática emitiu a artéria gastroduodenal, pancreaticoduodenal cranial e ramos hepáticos para o fígado. A artéria gastroduodenal lançou a artéria gastroepiplóica direita para a curvatura maior do estômago, a artéria gástrica direita para a curvatura menor do estômago, ramos esofágicos e pilóricos. A artéria pancreaticoduodenal cranial distribuiu-se para a porção inicial do duodeno e lobo direito do pâncreas.

A artéria celíaca, através de seus ramos, foi a artéria responsável pela irrigação do estômago, pâncreas, fígado, baço e porção inicial do duodeno.

\section{NOTAS INFORMATIVAS}

${ }^{1}$ Cristália-Produtos Químicos e Farmacêuticos Ltda, São Paulo, SP, Brasil.

${ }^{2}$ Liquemine-Roche, Rio de janeiro, RJ, Brasil.

\section{REFERÊNCIAS}

1 Barone R. 1996. Anatomie comparée des mammifères domestiques - Angiologie. 2éme edn. Paris: Vigot, 904p.

2 Cavalcante Filho M.F., Miglino M.A., Machado G.V., Bevilacqua E. \& Neves W.C. 1998. Estudo comparativo sobre o suprimento arterial do estômago do queixada (Tayassu pecari) e do cateto (Tayassu tajacu) [Linnaeus, 1789]. Brazilian Journal of Veterinary Research and Animal Science. 35(1): 20-24. 
3 Culau P.O.V., Azambuja R.C. \& Campos R. 2008. Ramos colaterais parietais e terminais da aorta abdominal em Myocastor coypus (nutria). Ciência Rural. 38(4): 997-1002.

4 Evans H.E. 1993. The heart and the arteries. In: Evans H.E. (Ed). Miller's Anatomy of the dog. 3rd edn. Philadelphia: W. B. Saunders Company, pp.586-681.

5 Ghoshal N.G. 1986. Coração e artérias do carnívoro. In: Getty R. (Ed). Anatomia dos animais domésticos. 5.ed. Rio de Janeiro: Interamericana, pp.1497-1550.

6 International Committee on Veterinary Gross Anatomical Nomenclature. 2005. Nomina anatomica veterinaria. 5 th edn. New York, 198p.

7 Lindemann T. 2002. Estudo da distribuição e dos territórios das artérias cerebrais rostral, média e caudal e cerebelares rostral, média e caudal na superfície do encéfalo em Didelphis albiventris (gambá). 154f. Porto Alegre, RS. Tese (Doutorado em Ciências Veterinárias) - Programa de Pós-graduação em Ciências Veterinárias, Universidade Federal do Rio Grande do Sul.

8 Machado G.V. 1999. Sobre a divisão do tronco celíaco e seus ramos no ouriço-cacheiro (Sphiggurus spp. Cuvier, 1825). In: Anais do VII Evento de Iniciação Científica da UFPR (Curitiba, Brasil). p.451.

9 Machado G.V., Gonçalves P.R., Parizzi A. \& Souza J.R. 2006. Padrão de divisão e distribuição das artérias mesentéricas no ratão-do-banhado (Myocastor coypus - Rodentia: Mammalia). Biotemas. 19(1): 59-63.

10 Machado G.V., Souza J.R., Gonçalves P.R., Parizzi A. \& Donin D.G. 2002. A artéria celíaca e seus ramos no ratão-do-banhado (Myocastor coypus - Rodentia: Mammalia). Biotemas. 15(2): 41-54. 
